\title{
Computation of the dynamic thermal dissipation properties of porous media by Brownian motion simulation: Application to an open-cell aluminum foam
}

\author{
Camille Perrot ${ }^{\mathrm{a})}$ \\ Groupe d'Acoustique de l'Université de Sherbrooke (GAUS), Department of Mechanical Engineering, \\ Université de Sherbrooke, Quebec JIK 2R1, Canada and Laboratoire des Sciences de l'Habitat (LASH), \\ ENTPE, DGCB URA CNRS 1652, rue Maurice Audin, 69518 Vaulx en Velin, France \\ Raymond Panneton \\ Groupe d'Acoustique de l'Université de Sherbrooke (GAUS), Department of Mechanical Engineering, \\ Université de Sherbrooke, Quebec J1K 2R1, Canada

\begin{abstract}
Xavier Olny
Centre d'Études Techniques de l'Équipement de Lyon, Département Villes et Territoires/Groupe AUE, 46 rue Saint Théobald, Bô̂te Postale 128, 38081 L'isle d'Abeau Cedex, France
\end{abstract}

(Received 7 May 2007; accepted 14 August 2007; published online 11 October 2007)

This paper reports simulation results of frequency dependent heat conduction through three-dimensional reconstructed unit cells of an open-cell aluminum foam under acoustic excitations. First, a three-dimensional random walk based algorithm is proposed to calculate the dynamic thermal permeability or dynamic bulk modulus of periodic complex porous geometries. Second, the error and convergence of the implemented calculation algorithm are quantified in terms of the random walk population, normalized trapping distance, and type of geometry. Finally, the algorithm is applied to the calculation of the dynamic bulk modulus of an aluminum foam and compared to laboratory measurements. Good agreement is obtained between simulations and measurements. () 2007 American Institute of Physics. [DOI: 10.1063/1.2786899]

\section{INTRODUCTION}

Can a periodic unit cell (PUC), whose microstructural parameters have been identified experimentally, serve as a basis to model acoustic dissipation phenomena in open cell foams? How do acoustic properties depend on microstructural parameters? These are two of the many questions that are dominating studies of relationships between microstructure and acoustic properties of porous media such as open cell foams.

Such questions may be addressed in different manners. A common method consists in conducting a lot of laboratory measurements on samples of varying microstructural parameters. ${ }^{1,2}$ Alternatively, in a search for a theoretical understanding, one may try to better understand the mathematical and physical basis of the macroscopic equations governing acoustic dissipation phenomena. ${ }^{3-7}$ Finally, numerical studies based on simulations can be considered. ${ }^{8-13}$ Each of these ways of considering these questions has advantages and disadvantages. Laboratory measurements are of indisputable values; however, their interpretation may be limited to a specific group of materials. Theoretical studies at the macroscopic scale are leading to robust models, but they also require measurements of nonindependent macroscopic parameters. Numerical simulations usually attempt to bridge the gap between theory and experiments. They are nevertheless typically restrained by either the need to simplify geometry, physics, or both.

\footnotetext{
${ }^{a)}$ Author to whom correspondence should be addressed. Electronic mail: camille.perrot@usherbrooke.ca
}

In recent years, another approach to the numerical study of acoustic properties of porous media has gained some interest. The idea is to numerically solve, in a microstructural configuration which consists of a PUC, the linearized Navier-Stokes equation in harmonic regime with the local incompressibility condition ${ }^{14}$ (viscous problem) and the linearized heat equation in harmonic regime ${ }^{7}$ (thermal problem), with appropriate boundary conditions, and then to study how volume-averaged properties of the velocity and thermal fields relate to microscopic details of the geometry. Compared to macroscopic models, such an approach offers the ability to study the microphysical basis of the acoustical macrobehavior. Additionally, we proceed by increasing order of complexity, by solving initially the thermal problem, scalar counterpart of the viscous one.

The dynamic thermal permeability $k^{\prime}(\omega)$ plays, in the description of the thermal exchanges between frame and saturating fluid, a role similar to the dynamic viscous permeability in the description of the viscoinertial forces. Following Lafarge et al., ${ }^{7}$ the thermal analog of the dynamic thermal permeability is defined by setting at any frequency $\Phi\langle\tau\rangle=\left[k^{\prime}(\omega) / \kappa\right] \partial\langle p\rangle / \partial t$, where $\Phi$ is the porosity of the porous media, $\langle\tau\rangle$ is the macroscopic excess temperature in air (the symbol \langle\rangle denotes an intrinsic air-phase average), $\kappa$ is the fluid thermal conductivity, and $\partial\langle p\rangle / \partial t$ is the macroscopic acoustic pressure derivative. $k^{\prime}(\omega)$ determination is important not only when a precise prediction of sound propagation and absorption through air-filled porous media is needed but also as a supplementary microstructure probe. Explicit relations leading to the propagation constant and the 
characteristic impedance of a medium from its dynamic viscous and thermal permeabilities can be found elsewhere. ${ }^{7}$ Remarkably, for the scalar problem the overall volume is equally taken into account in the definition of a pore radius, whereas in the viscous problem narrowing zones are clearly playing a particular role in the definition of an effective section.

For the case of the viscous problem, solutions by finite element methods (FEMs) have been investigated. Craggs and Hildebrandt ${ }^{8}$ solved the viscous problem for specific cross sections of uniform pores. Cortis et al. ${ }^{10}$ studied the case of a two-dimensional (2D) configuration made of a regular arrangement of solid cylinders. Gasser et al. ${ }^{13}$ treated the case of the face centered cubic sphere packing.

The random walk simulation method has been recently proposed by Lafarge ${ }^{15}$ to provide an efficient resolution of the thermal problem. The principle of the method consists in simulating the Brownian motion for a large number of the fluid-phase particles and to link their mean free paths to the thermal conduction properties of the confined fluid. ${ }^{16,17}$ An important point of the method is that, once the mean free path of a large number of particles has been estimated, the dynamic thermal response might be obtained for all frequencies. Contrary to finite element analysis, the solution has not to be computed at each frequency.

The random walk simulation method has been implemented in two and three dimensions for computing the trapping constant of a 2D arrangement of overlapping fibers of circular cross sections, ${ }^{17}$ and three-dimensional (3D) digitalized geometries. ${ }^{18}$ However, the trapping constant is only providing the asymptotic low frequency behavior of the thermal problem. The first numerical simulations in the harmonic regime have recently been proposed for the case of $2 \mathrm{D}$ regular and random arrangements of fibers with circular cross sections. $^{15}$

Broadly speaking, the literature on the numerical simulations of the dynamic thermal behavior of porous media has been focused on two- and three-dimensional arrangements of spheres. No numerical resolution of the thermal problem has been proposed for the case of an open-cell foam PUC. Moreover, the three-dimensional implementation of the random walk simulation method has been limited to the asymptotic low frequency behavior.

The aim of this study is to carry out a dynamic thermal permeability computation of a real open-cell foam. For that purpose, the random walk simulation method will be implemented in $3 \mathrm{D}$, for cellular periodic configurations, whose microstructural parameters have been experimentally identified by x-ray computed microtomography.

In Sec. II, the basic equations and relationships between the heat conduction and survival problems are recalled. Section III describes more specifically the Brownian motion simulation technique. Section IV treats the evaluation of the survival time and dynamic thermal permeability from the Brownian motion simulation technique. The convergence and error of the method are then exposed in Sec. V. Numerical and experimental results are presented and discussed in Sec. VI. Finally, in Sec. VII, we summarize and conclude this paper.

\section{BASIC EQUATIONS}

\section{A. Multiscale formulation}

At the microscopic scale, it has been shown by Lafarge et $a{ }^{7}{ }^{7}$ that the frequency dependent heat conduction boundary value problem is locally governed at order 0 by the linearized heat equation in the harmonic regime, the zero acoustic temperature at the solid interface $\partial \Omega$, and the periodicity condition on the volume $\Omega$. These equations are, respectively, given by

$$
\begin{aligned}
& \frac{j \omega}{\nu^{\prime}} \tau=\nabla^{2} \tau+\frac{j \omega p}{\kappa} \text { in } \Omega_{f}, \\
& \tau=0 \text { on } \partial \Omega, \\
& \text { periodicity of } \tau \text { and } p \text { in } \Omega,
\end{aligned}
$$

where $\nabla^{2}$ is the Laplace operator, $\tau$ and $p$ are the acoustic temperature and pressure, respectively, $\nu^{\prime}=\kappa / \rho_{0} C_{p}$ is the fluid thermal diffusivity, and $\rho_{0} C_{p}$ characterizes the fluid thermal inertia given by the product of the fluid density at equilibrium by its specific heat at constant pressure. Thus, $j \omega p / \kappa$ appears as the source term of the partial differential equation, $\nabla^{2} \tau$ as the diffusion term by thermal conduction in the fluid space, and $\left(j \omega / \nu^{\prime}\right) \tau$ the term of thermal inertia.

Note that $\Omega$ needs to be rhombohedral, so that boundaries are periodic. Clearly, this is an attempt to directly model the geometry of the porous structure by defining a PUC that captures the intricate details of the foam. As a result, this models an infinitely large matrix as if the porous structure within the unit cell were replicated in all other unit cells, without discontinuities in the porous structure between one unit cell and the one adjacent. It can be proved from homogenization techniques ${ }^{19}$ that this approach is valid if and only if the fundamental condition of scale separation is respected, that is, $l / L \ll 1$, where $l$ is the characteristic dimension of the heterogeneities, $L$ is the macroscopic characteristic dimension, and $l / L$ is a measure of the separation of scales. This means that the microscopic characteristic length must be small compared to the macroscopic sample size and to the physical phenomenon characteristic size. The macroscopic characteristic length $L$ is thus the smallest between the characteristic sample size and physical phenomenon. In acoustics, the physical phenomenon characteristic size is on the order of $\lambda / 2 \pi$, where $\lambda$ is the wavelength of the incident acoustic wave. Another important consequence of this scale separation hypothesis justified by homogenization techniques is that the pressure term $p$ appears as a spatial constant at the cell size level.

Under harmonic excitations, the fluid is locally subjected to a compression/expansion cycle resulting in a locally increasing/decreasing temperature. The local heat excess/ defect is consequently diffusing according to two antagonist dissipative mechanisms by thermal conduction and inertia depending on the angular frequency of excitation. The solidfluid interface temperature is assumed to be constant since the thermal inertia of the solid phase is generally much larger than the fluid one. This can be written by the condition (1 $-\Phi)\left(\rho_{0} C_{p}\right)_{\text {solid }} \gg \Phi\left(\rho_{0} C_{p}\right)_{\text {fluid. }}$. For the specific case of an alu- 
minum foam with a porosity of 0.92, (1- $\Phi)$ $\times\left(\rho_{0} C_{p}\right)_{\text {aluminum }} / \Phi\left(\rho_{0} C_{p}\right)_{\text {air }} \approx 170$ and the previous inequality is fully respected.

At the macroscopic scale, the overall dissipative phenomena by thermal effects averaged over an elementary $\Omega$-periodic volume are taken into account by its equivalent dynamic thermal permeability,

$$
\widetilde{k}_{\mathrm{eq}}^{\prime}(\omega)=\langle\tau\rangle \frac{\kappa}{j \omega p}
$$

whose expression is defined by the product of the mean acoustic temperature field and the inverse of the source term.

Here the equivalent dynamic thermal permeability of the porous aggregate is linked to the dynamic thermal permeability of the fluid phase $\widetilde{k}^{\prime}(\omega)$ (as defined by Lafarge et al. ${ }^{7}$ ) by the relation $\widetilde{k}_{\mathrm{eq}}^{\prime}(\omega)=\widetilde{k}^{\prime}(\omega) / \Phi$.

From then on, the computation of the dynamic thermal permeability is clearly based on the resolution of the local problem [Eqs. (1)-(3)] and the spatial integration of the solution temperature field.

\section{B. Analogy with the diffusion-controlled trapping problem}

Consider the steady-state problem of diffusion and reaction among absorbing "traps" in which the concentration field $n$ of the reactants in the air-filled region is governed by

$$
\frac{\kappa_{b}}{D} n=\nabla^{2} n+\frac{\sigma}{D} \quad \text { in } \Omega_{f},
$$

with the boundary condition at the pore-trap interface given by

$$
n=0 \quad \text { on } \partial \Omega \text {, }
$$

indicating that the process is diffusion controlled, i.e., traps are perfect absorbers, and the periodicity condition

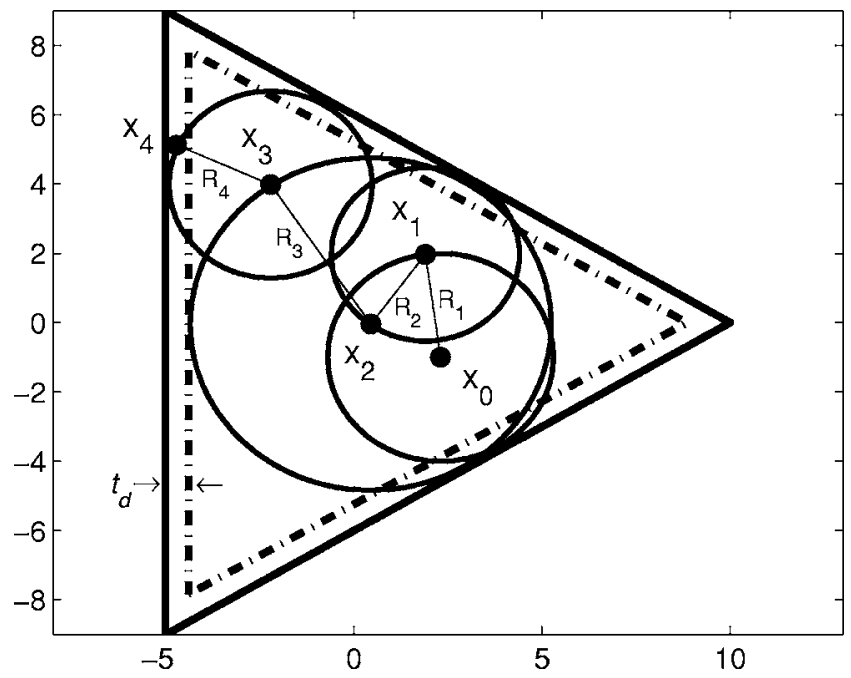

FIG. 1. Two-dimensional schematic representation of the construction of a random walk in a hypothetical triangular cell. periodicity of $n$ in $\Omega$.

Here, $D$ is the diffusion coefficient of the reactant, $\kappa_{b}$ is an air-filled reaction term associated with the decay of physical quantities such as concentration field, and $\sigma$ is a prescribed rate of production of the reactants per unit pore volume.

Note that the frequency dependent heat conduction problem [Eqs. (1)-(3)] is formally identical to the steady-state survival problem [Eqs. (5)-(7)], according to the following identifications:

$$
\begin{aligned}
& n=\tau, \\
& \frac{\kappa_{b}}{D}=\frac{j \omega}{\nu^{\prime}},
\end{aligned}
$$

and

$$
\frac{\sigma}{D}=\frac{j \omega p}{\kappa} .
$$

This analogy is used to write the thermal permeability in terms of the steady-state survival problem variables,

$$
\tilde{k}_{\mathrm{eq}}^{\prime}(\omega)=D \frac{\langle n\rangle}{\sigma}
$$

In this regime, the amount of diffusing species $\sigma$ created per unit time and air volume is exactly compensated by those absorbed on contact with the pore walls and in the air-filled region. Thus, the mean field $\langle n\rangle$ must be equal to the product $\sigma\langle t\rangle$, where $\langle t\rangle$ is the mean survival time of one diffusing particle which is released at random position in the pore space. From that, one gets the following equality:

$$
\langle t\rangle=\frac{\langle n\rangle}{\sigma} .
$$

Substituting Eq. (12) into Eq. (11) yields

$$
\tilde{k}_{\mathrm{eq}}^{\prime}(\omega)=D\langle t\rangle .
$$

It makes clearly apparent from relation (13) that numerical methods usually dedicated to diffusion problems of a solute in stationary regime [Eqs. (5)-(7)] can be used to solve the analog frequency dependent heat conduction problem [Eqs. (1)-(3)].

\section{BROWNIAN MOTION SIMULATION}

\section{A. Construction of a random walk}

The basic idea of the employed simulation method ${ }^{17,20}$ is that the zigzag random motion of the diffusing particle need not be simulated in detail; instead, it is taken into account in a single simulation step using the first passage time probability distribution, ${ }^{21}$

$$
P(t, R)=1+2 \sum_{m=1}^{\infty}(-1)^{m} \exp \left(-\frac{D m^{2} \pi^{2} t}{R^{2}}\right),
$$

where $P(t, R)$ is the cumulative distribution function associated with the time $t$ taken for a random walker initially at the origin to reach the surface of the sphere of radius $R$ in the absence of air-filled absorption. Consequently, one constructs 


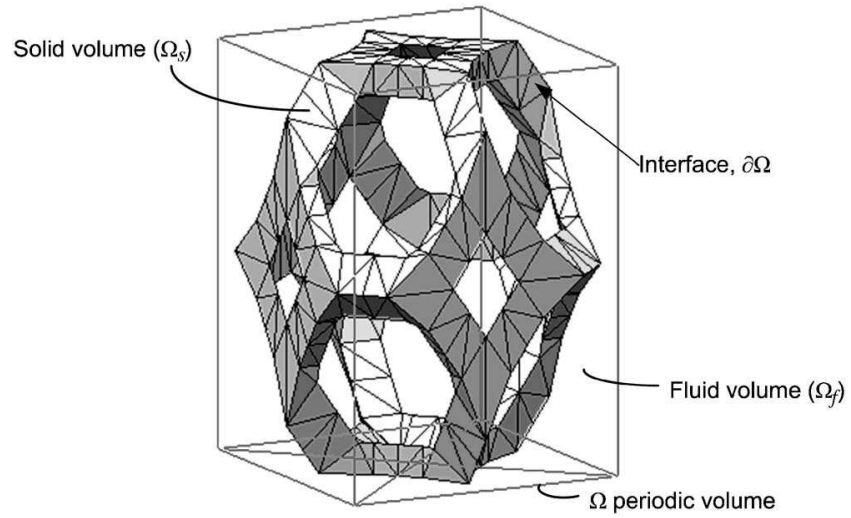

FIG. 2. An idealized three-dimensional periodic unit cell (3D PUC) of an open-cell aluminum foam whose characteristic dimensions have been identified experimentally by computed microtomography (Ref. 26). The filiform box defines the periodic control volume for the random walk simulations.

the largest concentric sphere of radius $R_{i}$, which does not overlap with the solid phase acting as a trap. The next position of the diffusing particle, the walker, is taken randomly on the concentric surface sphere. This process is repeated until the random walker gets trapped (i.e., it comes within a trapping distance $t_{d}$ of a trap).

The employed simulation method relies consequently on the construction of a random walk, as illustrated in Fig. 1. The initial random walker position $x_{0}$ is taken randomly in the fluid space, schematically represented by a triangular prism in Fig. 1. The largest concentric sphere of radius $R_{1}$ is constructed, without overlapping with the solid phase. The next position $x_{1}$ of the walker is determined randomly at the surface of the sphere of center $x_{0}$ and radius $R_{1}$. In this manner, a Brownian motion path $x_{0}, x_{1}, \ldots, x_{n}$ is generated and stopped when $x_{n}$ comes within a small distance $t_{d}$ of the solid trap $\left(R_{n+1}<t_{d}\right)$.

\section{B. Random walk algorithm}

Now that the construction principle of a random walk has been recalled, the employed algorithm is briefly presented. It is a purely geometrical problem solved into six different steps. (1) Randomly position a walker into an $\Omega$-periodic volume [e.g., a rectangular prism defined by the coordinates $\left(x_{\min }, x_{\max }\right),\left(y_{\min }, y_{\max }\right),\left(z_{\min }, z_{\max }\right)$ for a threedimensional PUC-see Fig. 2]. (2) Find the concentric sphere of largest radius $R$ which does not overlap any solid surface. (3) Check if the walker positioned at point $M$ lies inside the fluid volume $V_{f}$. If not, return to step 1. Else, increment radius counter $n$ and go to step 4. (4) Check if the walker is trapped. If $R<t_{d}$, the walker is trapped, go to step 1. Else, if the walker is not trapped, go to step 5. (5) Randomly position the walker at the surface of the sphere of radius $R$. (6) Apply a periodic boundary condition. If the random walker lies outside $\Omega$, then it must be translated by a period of $\Omega$ in order to be in an equivalent position than the one it would be if the porous media were infinite. Else, the walker position stays unchanged. Then, go to step 2 until the walker is trapped. Once the random walker is trapped, the radius counter is reinitialized to zero and a new random

\section{Slits \\ width $w$ \\ $\Lambda^{\prime}=w=R$}

Circle

radius $R$

$\Lambda^{\prime}=R$

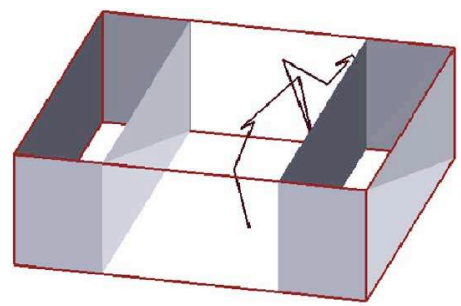

\section{Rectangle}

Sides $2 a$ and $2 b$

$\Lambda^{\prime}=a=b=R / \sqrt{ } 2$

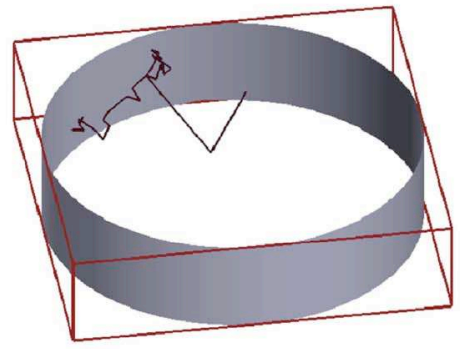

\section{Equilateral triangle}

side $s$

$\Lambda^{\prime}=s / 2 \sqrt{3}=R / 2$
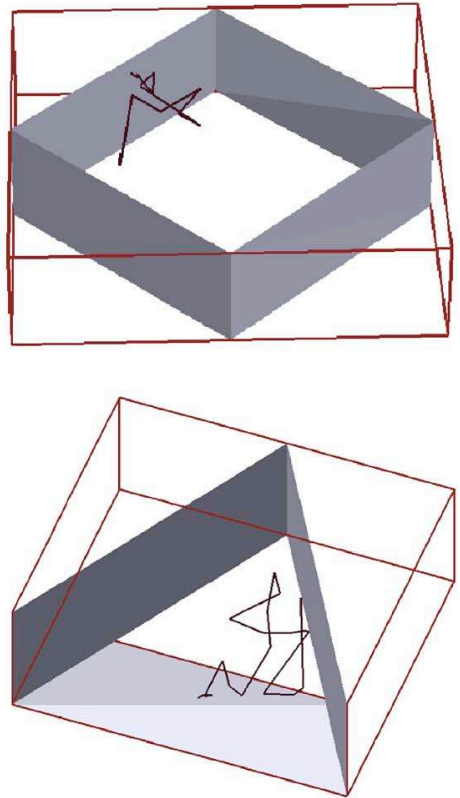

FIG. 3. (Color online) Synthesis of approximated analytical reference solutions available for specific cross-sectional shapes of uniform pores. These solutions are valid in two or three dimensions. The filiform boxes define the control volume for the random walk simulations.

walker can be started from step 1. A full description of the random walk algorithm implementation in three-dimensional PUCs can be found elsewhere. ${ }^{22}$

Once the random walk algorithm has been implemented, numerical simulations can be carried out for a geometric configuration (e.g., the three-dimensional PUC shown in Fig. 2). For $N$ random walk simulations, collected data consist in $N$ successions of radius. The question is then to know how these collections of random walks and radius can be translated in terms of the dynamic thermal permeability.

\section{CALCULATION OF DYNAMIC THERMAL PERMEABILITY}

Given the probability $p(R)$ of crossing for the first time the surface of a sphere of radius $R$ and the time $t(R)$ for 
trapping or crossing for the first time the surface of this sphere, the average time for trapping a given random walker along the path $x_{0}, x_{1}, \ldots, x_{n}$ is simply given by

$$
\begin{aligned}
t_{i}\left(x_{0}, \ldots, x_{n}\right)= & t\left(R_{1}\right)+p\left(R_{1}\right) t\left(R_{2}\right)+p\left(R_{1}\right) p\left(R_{2}\right) t\left(R_{3}\right) \\
& +\cdots+p\left(R_{1}\right) \cdots p\left(R_{n-1}\right) t\left(R_{n}\right) .
\end{aligned}
$$

For a large number $N$ of random initial positions $x_{0}$ (or random walk simulations) in the porous space, the mean survival time $\langle t\rangle$ of a particle is the mean survival time of $N$ random walks,

$$
\langle t\rangle=\frac{1}{N} \sum_{i=1}^{N} t_{i}\left(x_{0}, \ldots, x_{n}\right) .
$$

Once the collection of radius have been determined by random walk simulations, the dynamic thermal permeability determination $\widetilde{k}_{\mathrm{eq}}^{\prime}(\omega)=D\langle t\rangle$ requires the knowledge of two functions of $R, p(R)$, and $t(R)$, whose expressions are given below.

\section{A. First passage time probability}

In the presence of air-filled absorption, the probability $p(R)$ that a walker initially at the center of a sphere survives and reaches the boundary is not unity and becomes

$$
p(R)=\int_{0}^{\infty} \frac{\partial P(t, R)}{\partial t} \exp \left(-\kappa_{b} t\right) d t,
$$

where $\kappa_{b}$ is the air-filled absorption coefficient, which gives in two and three dimensions, ${ }^{23}$

$$
p(R)_{2 \mathrm{D}}=\frac{1}{I_{0}(\mu)}
$$

and

$$
p(R)_{3 \mathrm{D}}=\frac{\mu}{\sinh (\mu)},
$$

respectively. In these equations, $I_{0}$ is the modified Bessel function of the first kind and zero order, and

$$
\mu=R \xi
$$

with

$$
\xi=\sqrt{\frac{\kappa_{b}}{D}} .
$$

\section{B. Survival time}

In a stationary regime, a particle released at radius $r$, diffusing with the diffusion coefficient $D$ and being absorbed either instantly at $r=R$ or in the air-filled region (given an air-filled absorption coefficient $\kappa_{b}$ ), has a survival time $t(r)$ given by ${ }^{24,25}$

$$
t(r)=u_{R}(r) / D,
$$

where $u_{R}(r) \equiv n(x, y, z)$ is the scaled concentration field which verifies Eq. (5) in domain $x^{2}+y^{2}+z^{2}<R^{2}$, boundary condition $u_{R}(r=R)=0$, and condition $\sigma=D$ (the source term is set to unity),

$$
\begin{aligned}
& \frac{\kappa_{b}}{D} u_{R}(r)=\nabla^{2} u_{R}(r)+1, \\
& \left.u_{R}\right|_{r=R}=0 .
\end{aligned}
$$

The three-dimensional solution of this boundary value problem [Eqs. (23) and (24)] is derived in spherical coordinates and yields

$$
u_{R}(r)=\frac{1}{\xi^{2}}\left[1-\frac{j_{0}(j \xi r)}{j_{0}(j \xi R)}\right],
$$

where $j_{0}$ denotes the spherical Bessel function of the first kind of order 0 .

Then, the average time for a particle released at the origin to be either absorbed in the air-filled region or to be present for the first time at $r=R$ can be defined from Eq. (22) by

$t(R)=u_{R}(0) / D$,

where, from Eq. (25),

$$
u_{R}(0)=\frac{1}{\xi^{2}}\left[1-\frac{1}{j_{0}(j \xi R)}\right],
$$

because $j_{0}(0)=1$.

\section{Dynamic thermal permeability}

Now, going back to the original frequency dependent heat conduction problem, making use of the analogy given in Eq. (9) and substituting the canonical field for the scaled field $u_{R}(0)$, Eq. (27) rewrites

$$
\tilde{u}_{R}(0)=-\frac{1}{K^{2}}\left[1-\frac{1}{j_{0}(K R)}\right],
$$

with

$$
K=\left(\frac{-j \omega}{\nu^{\prime}}\right)^{1 / 2} .
$$

From Eqs. (13) and (26), the dynamic thermal permeability simplifies to

$$
\tilde{k}_{\mathrm{eq}}^{\prime}(\omega)=\left\langle\tilde{u}_{R}(0)\right\rangle .
$$

For the sake of simplicity, the notation $\tilde{u}_{R}(0)$ is replaced by $\tilde{u}(R)$ and

$$
\tilde{k}_{\mathrm{eq}}^{\prime}(\omega)=\langle\widetilde{u}(R)\rangle=\frac{1}{N} \sum_{i=1}^{N} \tilde{u}_{i}\left(x_{0}, \ldots, x_{n}\right),
$$

with, for a given random walk,

$$
\begin{aligned}
\tilde{u}_{i}\left(x_{0}, \ldots, x_{n}\right)= & \tilde{u}\left(R_{1}\right)+p\left(R_{1}\right) \tilde{u}\left(R_{2}\right)+p\left(R_{1}\right) p\left(R_{2}\right) \tilde{u}\left(R_{3}\right) \\
& +\cdots+p\left(R_{1}\right) \cdots p\left(R_{n-1}\right) \tilde{u}\left(R_{n}\right),
\end{aligned}
$$

where the first passage time probability in the presence of air-filled absorption $p(R)$ is given by Eqs. (18) and (19) in $2 \mathrm{D}$ and $3 \mathrm{D}$, respectively, and the scaled canonical field $\widetilde{u}(R)$ is given by Eq. (28) in 3D. In 2D, Eq. (28) has to be modified by simply substituting $J_{0}$ for $j_{0}$, where $J_{0}$ is the Bessel function of the first kind and order 0. 

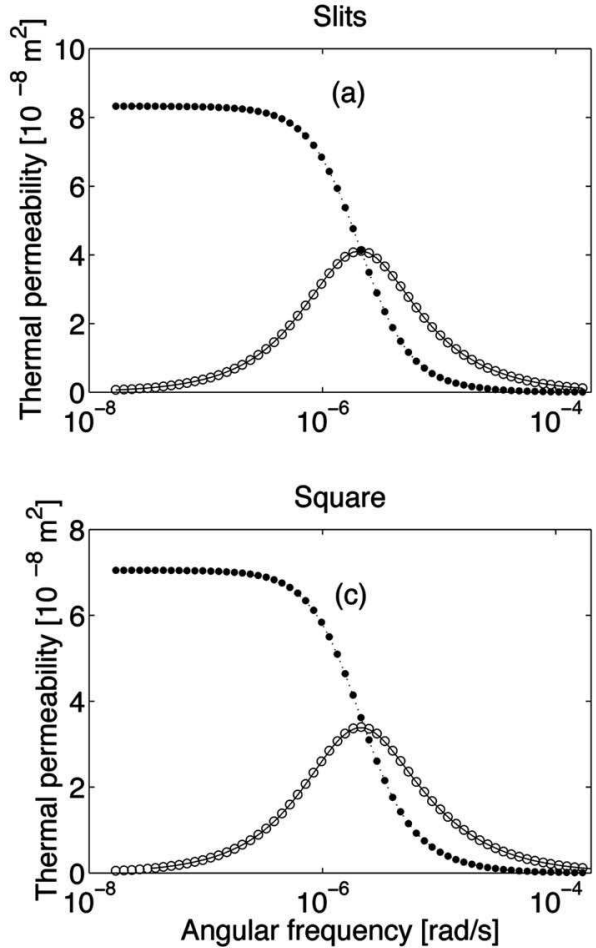
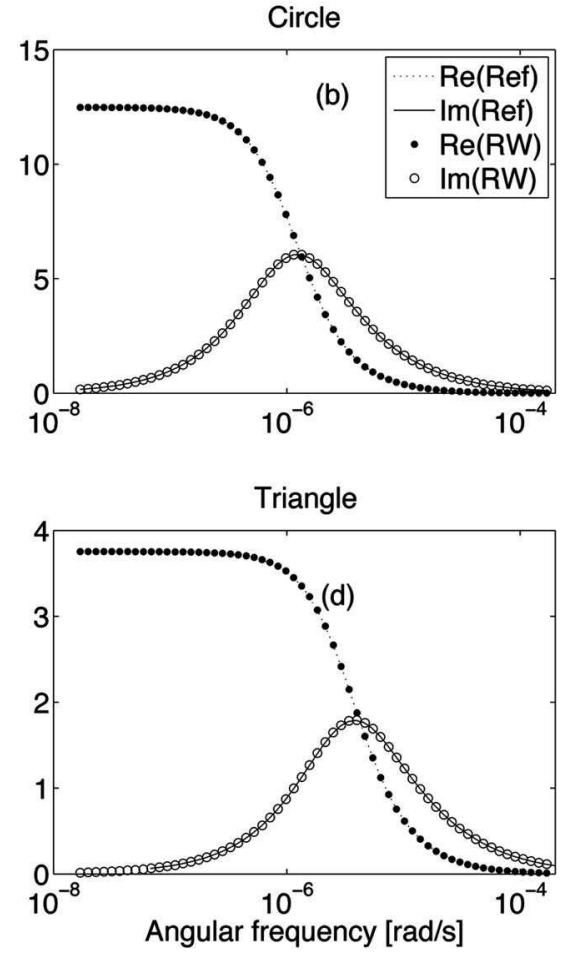

FIG. 4. Comparison between analytical reference solutions (Ref) and numerical computations performed by means of the three-dimensional implementation of the random walk (RW) algorithm for a number of random walks $N=500000$ and a normalized trapping distance $\delta=10^{-5}$ (the circumscribed radius of the specific crosssectional shapes of uniform pores has been fixed to $R=1 \mathrm{~mm}$ ).
The interest of this somewhat unconventional method is that the simulation of $N$ random walks is independent of frequency. This means that, once the $N$ successions of radius are known for a given geometric configuration, the thermal permeability can be computed whatever the frequency, with the same computational cost. For this reason, the difficulties met with conventional computational methods, such as the finite element method, are overcome. With the finite element method, the mesh must be refined in the vicinity of the interface when the frequency is increased in order to take into account the decreasing thickness of the boundary layer; this may rapidly become prohibitive in terms of computation time and memory allocation for three-dimensional configurations.

\section{CONVERGENCE AND ERROR OF THE METHOD}

A simulation procedure is now proposed to test the convergence of the numerical solution and to estimate the error made on the prediction. In this proposed simulation procedure, the dynamic thermal permeability is computed on 60 frequency points uniformly distributed on a logarithmic scale
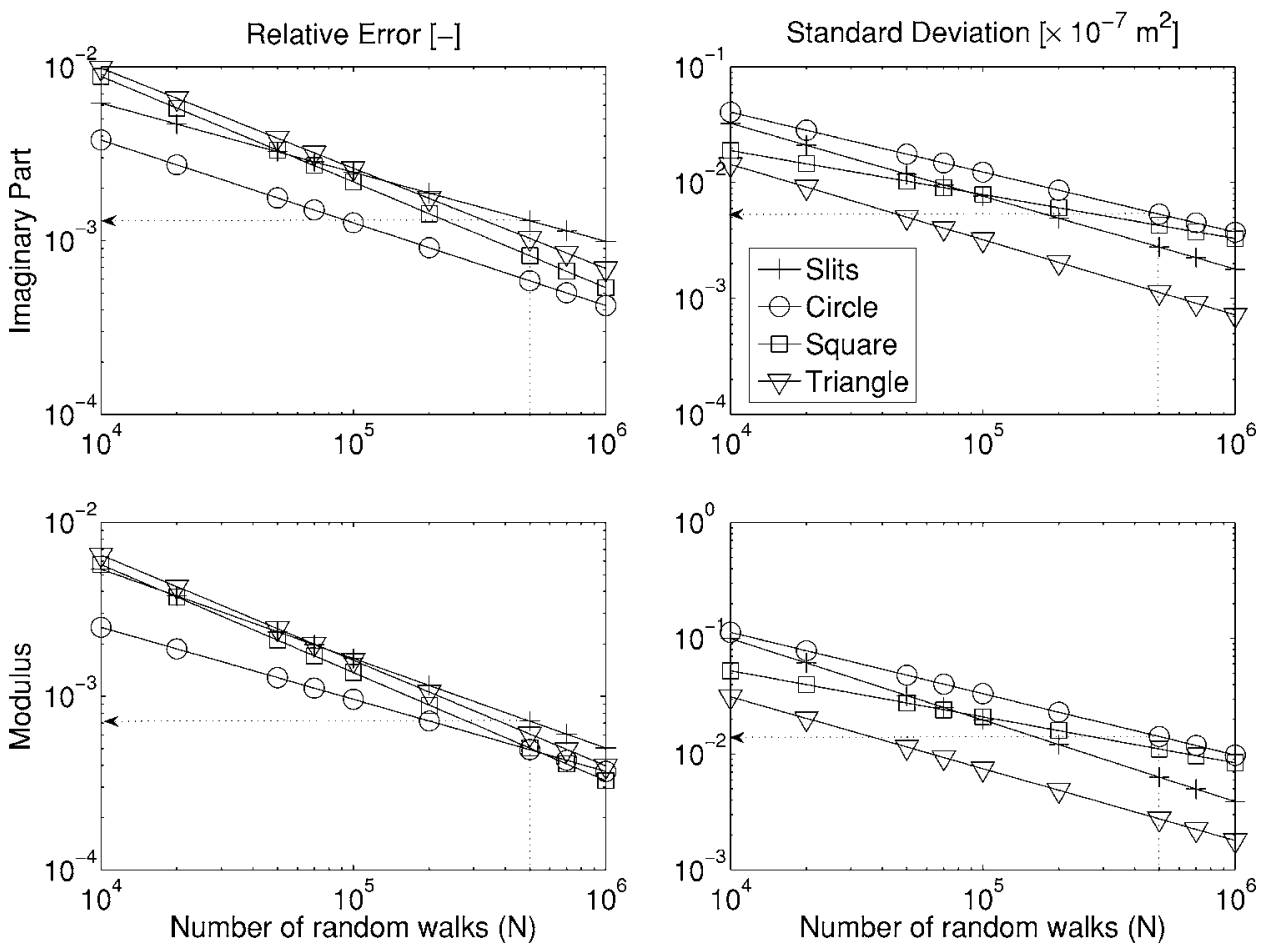

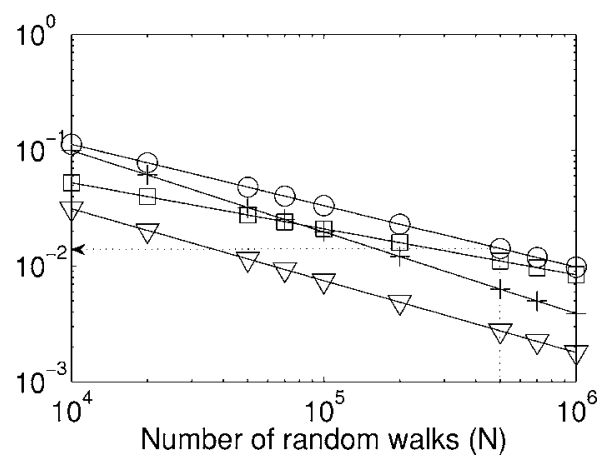

FIG. 5. Mean relative error and mean standard deviation on the dynamic thermal permeability with the number of random walks, in imaginary part and modulus. In this example, the normalized trapping distance $\delta$ has been fixed to $10^{-5}$. 
TABLE I. Normalized trapping distances $\delta$ relative to the highest correlation coefficients between the computed mean relative errors $(\varepsilon)$ of the predicted dynamic thermal permeabilities and a function of the form $\log 10(\varepsilon)=a \log 10(N)+b$, where $N$ is the number of random walks.

\begin{tabular}{cccc}
\hline \hline Geometry & Slits, square & Circle & Triangle \\
\hline$\delta=t_{d} / R$ & $10^{-4}$ & $10^{-5}$ & $10^{-6}$ \\
\hline \hline
\end{tabular}

and centered around $\omega_{\mathrm{tc}}$ - the critical angular frequency for thermal effects (characterizing the transition from the low frequency isothermal regime to the high frequency adiabatic regime). For the case of a circular cross-section tube, the critical thermal angular frequency is given by the following relationships: ${ }^{7}$

$$
\omega_{\mathrm{tc}}=\frac{\Phi \nu^{\prime}}{k_{0}^{\prime}},
$$

with

$$
k_{0}^{\prime}=\frac{\Phi \Lambda^{\prime 2}}{8},
$$

where $\Phi$ is the open porosity, $k_{0}^{\prime}$ is the static thermal permeability or low frequency limit of the dynamic thermal permeability, and $\Lambda^{\prime}=2 V_{p} / S_{p}$ is the thermal characteristic length ${ }^{4}$ defined as twice the ratio between the pore volume $V_{p}$ and wet surface $S_{p}$ which generalizes the notion of hydraulic radius for arbitrary geometries of the frame. This allows estimating a priori the critical thermal angular frequency from the knowledge of the fluid physical characteristics $\left(\nu^{\prime}\right.$ $=\kappa / \rho_{0} C_{p} \approx 2 \times 10^{-5} \mathrm{~m}^{2} \mathrm{~s}^{-1}$ for air) and pore geometry $(\Phi$ and $\Lambda^{\prime}$ are computed from spatial integration on the fluid volume and wet surface of the PUC) and thus deducing the angular frequencies for which the dynamic thermal permeability will be computed.
In order to simulate a large number of random walks for evaluating the influence of the simulation parameters on the dynamic thermal permeability results, simulations of random walks will be carried out on a personal computer (PC) cluster using 846 nodes thanks to the Scientific Computation Center facility of the Université de Sherbrooke [each computational unit is composed of an Intel P4 processor running at $3.2 \mathrm{GHz}$ with a 2 Go random access memory (RAM)].

\section{A. Solution convergence}

In the general case made of a nontrivial geometric configuration, there is no reference solution to test the solution convergence. However, the solution stability can be studied by means of indicators called norms. For this purpose, the evolution of the relative difference between the computed response for a number of random walks equal to $N$ and the one computed for a number of random walks equal to $N$ $+\Delta N$ will be examined. In practice, $\Delta N$ is fixed to 2000 . Two norms are presented below.

The geometric configuration studied is a typical idealized foam microstructure, a three-dimensional PUC whose characteristic dimensions have been identified by computed microtomography ${ }^{26}$ (i.e., an orthotropic tetrakaidecahedron having ligaments of triangular cross-section shapes, see Fig. 2).

\section{1. $L_{2}$ norm}

$L_{2}$ norm, as formulated here, is a measurement of the solution stability with respect to the number of random walks. It represents the sum of the relative variations between the computed response for a number of random walks equal to $N+\Delta N$ and the computed response for a number of random walks equal to $N$. It is given by

$$
L_{2}=\sqrt{\frac{\sum_{\omega}\left\{\operatorname{Re}\left[\tilde{k}_{\mathrm{eq}}^{\prime}(\omega)_{N+\Delta N}\right]-\operatorname{Re}\left[\tilde{k}_{\mathrm{eq}}^{\prime}(\omega)_{N}\right]\right\}^{2}}{\sum_{\omega}\left\{\frac{\operatorname{Re}\left[\tilde{k}_{\mathrm{eq}}^{\prime}(\omega)_{N+\Delta N}\right]+\operatorname{Re}\left[\tilde{k}_{\mathrm{eq}}^{\prime}(\omega)_{N}\right]}{2}\right\}^{2}}+\frac{\sum_{\omega}\left\{\operatorname{Im}\left[\tilde{k}_{\mathrm{eq}}^{\prime}(\omega)_{N+\Delta N}\right]-\operatorname{Im}\left[\tilde{k}_{\mathrm{eq}}^{\prime}(\omega)_{N}\right]\right\}^{2}}{\sum_{\omega}\left\{\frac{\operatorname{Im}\left[\tilde{k}_{\mathrm{eq}}^{\prime}(\omega)_{N+\Delta N}\right]+\operatorname{Im}\left[\tilde{k}_{\mathrm{eq}}^{\prime}(\omega)_{N}\right]}{2}\right\}^{2}}},
$$

where Re and Im denotes the real and imaginary parts of a complex number.

\section{2. $L_{\infty}$ norm}

$L_{\infty}$ norm is a measurement a priori more constraining than $L_{2}$ norm because, this time, it is the largest of the local differences (at a given angular frequency) between the com- puted response for a number of random walks equal to $N$ $+\Delta N$ and the computed response for a number of random walks equal to $N$, which is compared to the averaged response. Contrary to $L_{2}$ norm, the cumulated frequency response fluctuations are no more smoothed by the averaged cumulated response. In addition, the response is forced to be computed on a sufficiently large number of random walks for the largest relative fluctuations to be lower than a specific threshold value. It is given by 


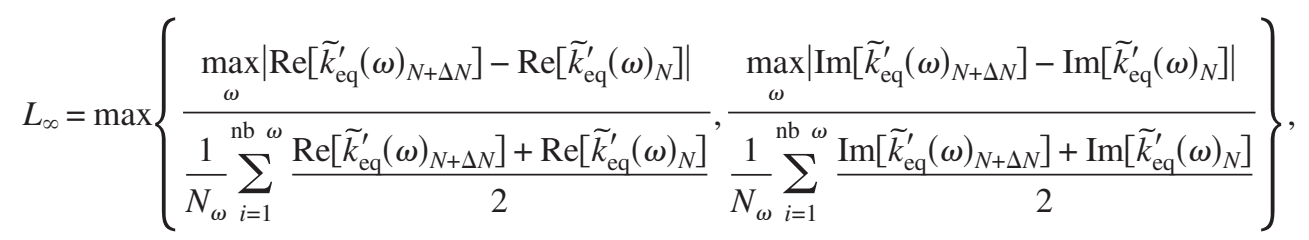

where $N_{\omega}$ is the number of angular frequency for which the dynamic thermal permeability is computed.

\section{B. Error estimation}

The knowledge of reference solutions enables one to estimate the errors made on the numerical predictions as a function of the simulation parameters. Here, existing reference solutions for the case of specific cross-sectional shapes of uniform pores are first recalled. Second, a straightforward logarithmic law relating the error evolution with the number of random walks is proposed from simple considerations on stochastic phenomena. As a result of the simulations, the relevance of such a law can be evaluated in a least squares sense from the examination of the determined correlation coefficients. Information about the error decay rate with the number of random walks, for different trapping distances, can then be derived from the coefficients of the logarithmic regressions.

\section{Reference solutions}

Stinson $^{27}$ proposes simplifying the exact Kirchhoff theory ${ }^{28}$ on the propagation of sound in circular tubes for the range of radii and frequencies defined by $r f^{3 / 2}<10^{4} \mathrm{~m} \mathrm{~s}^{-3 / 2}$ and $r>10 \mu \mathrm{m}$. For the thermal problem, the author seeks approximate solutions for arbitrary shapes of uniform pores expressed in terms of a single function $F\left(\nu^{\prime}\right)$. Hence, the dynamic thermal permeability can be written in the following general form:

$$
k^{\prime}(\omega)=\frac{\nu^{\prime}}{j \omega} F\left(\nu^{\prime}\right) .
$$

Specific cross-sectional shapes that were considered are shown in Fig. 3 with $\Lambda^{\prime}$ expressed as a function of their geometric descriptors. Solution for a slit of width $w$,

$$
F\left(\nu^{\prime}\right)=1-\frac{\tanh \left(\frac{w}{2} \sqrt{\frac{j \omega}{\nu^{\prime}}}\right)}{\frac{w}{2} \sqrt{\frac{j \omega}{\nu^{\prime}}}},
$$

was discussed by Attenborough. ${ }^{29}$ The result for a circular tube of radius $R$ which was originally obtained by Zwikker and $\operatorname{Kosten}^{30}$ is expressed by

$$
F\left(\nu^{\prime}\right)=1-\frac{2}{R \sqrt{\frac{-j \omega}{\nu^{\prime}}}} \frac{J_{1}\left(R \sqrt{\frac{-j \omega}{\nu^{\prime}}}\right)}{J_{0}\left(R \sqrt{\frac{-j \omega}{\nu^{\prime}}}\right)},
$$

where $J_{1}$ is the Bessel function of the first kind and order 1 . Stinson ${ }^{27}$ derived the solutions for a rectangular cross section,

$$
F\left(\nu^{\prime}\right)=\frac{4 j \omega}{\nu^{\prime} a^{2} b^{2}} \sum_{k=0}^{\infty} \sum_{n=0}^{\infty}\left[\alpha_{k}^{2} \beta_{n}^{2}\left(\alpha_{k}^{2}+\beta_{n}^{2}+\frac{j \omega}{\nu^{\prime}}\right)\right]^{-1},
$$

where the coefficients $\alpha_{k}$ and $\beta_{n}$ are given by

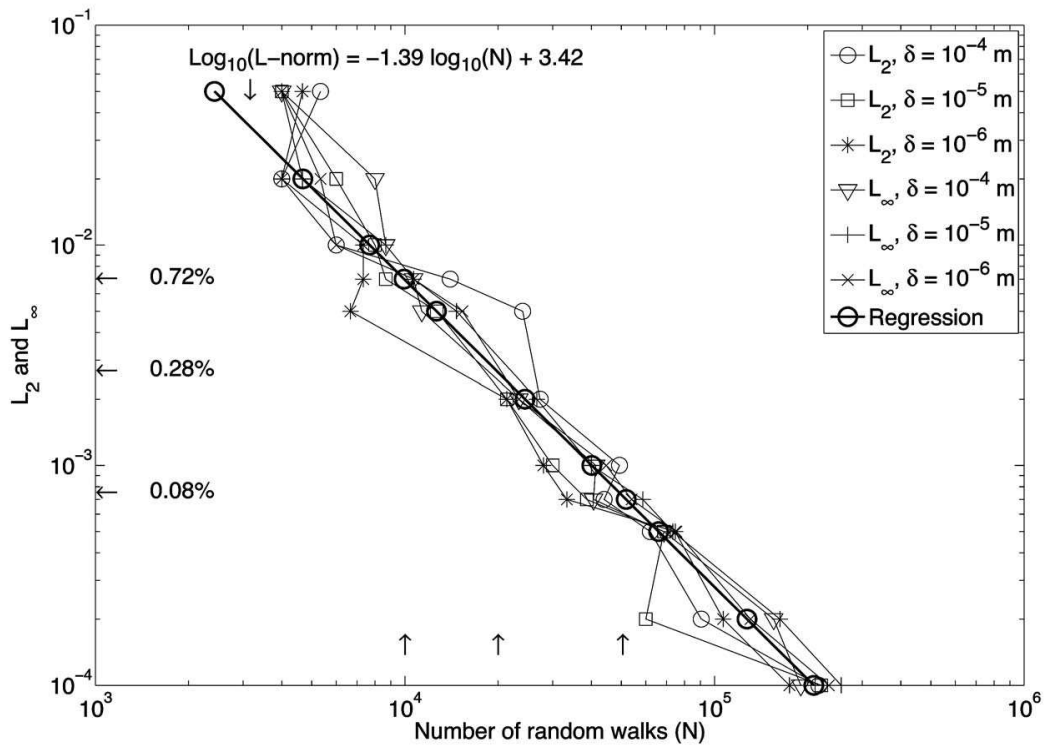

FIG. 6. Convergence in $L_{2}$ and $L_{\infty}$ norms for different normalized trapping distances $\delta$ on a periodic unit cell (PUC) geometry. 

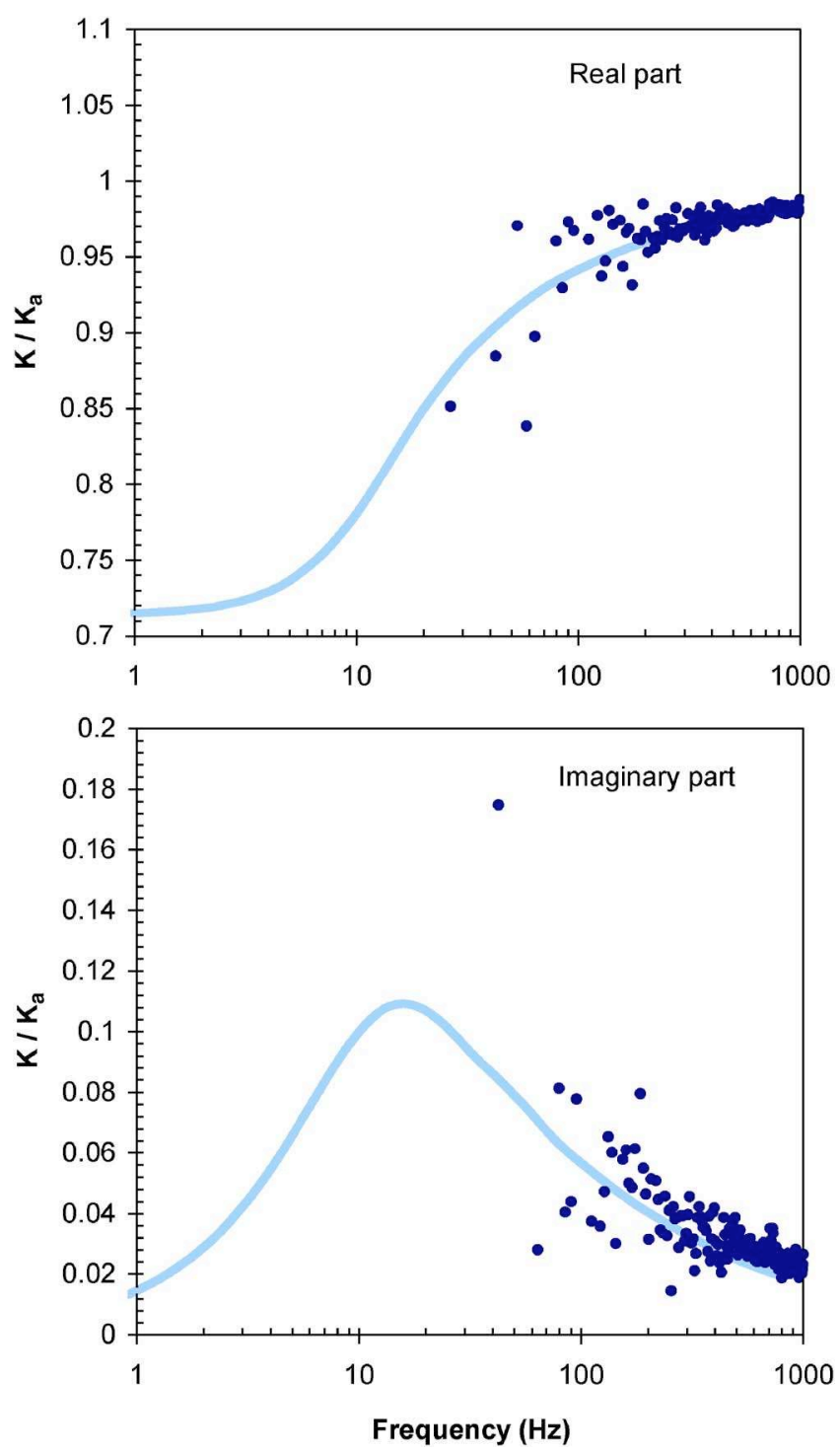

FIG. 7. (Color online) Dynamic bulk modulus $\widetilde{K}_{\text {eq }}$ normalized by the adiabatic bulk modulus $K_{a}$. Comparison between impedance tube measurements $(\bullet \bullet)$ and the random walk simulation method applied on the identified 3D PUC (Ref. 26) (一).

$$
\alpha_{k}=\left(k+\frac{1}{2}\right) \frac{\pi}{a}, \quad \beta_{n}=\left(n+\frac{1}{2}\right) \frac{\pi}{b} .
$$

Finally, the derivation of $F\left(\nu^{\prime}\right)$ for the equilateral triangle is due to Stinson and Champoux ${ }^{31}$ who found

$$
F\left(\nu^{\prime}\right)=\frac{\varepsilon^{2}-3 \varepsilon \operatorname{coth}(\varepsilon)+3}{\varepsilon^{2}},
$$

with

$$
\varepsilon=\frac{s \sqrt{3}}{4} \sqrt{\frac{j \omega}{\nu^{\prime}}} .
$$

\section{Error evolution law}

Stochastic phenomena are generally characterized by an error $\varepsilon$ inversely proportional to the root of the number of experiments $N .{ }^{16}$ If this tendency holds for the Brownian motion simulation problem, it means that it is necessary to increase the number of random walks by two orders of magnitude with a view to reducing the error on the estimate by one order of magnitude. Therefore, it is important to use a dynamic scale providing an identical sensitivity on several orders of magnitude. By taking the decimal logarithm of such a tendency, one can propose the following error evolution law:

$$
\log _{10} \varepsilon=a \log _{10} N+b,
$$

where $a$ is a linear decay rate expressed in (number of random walks) $)^{-1}$ and $b$ is the initial error (dimensionless) obtained for a unitary number of random walks. Following these observations, it is proposed to compute these coefficients by means of linear regressions in the least squares sense and to evaluate the linear correlation hypothesis between variables $\log _{10} \varepsilon$ and $\log _{10} N$ for different geometries and trapping distances.

\section{RESULTS AND DISCUSSION}

In this section, the proposed simulation method is applied to canonical geometries and to an idealized PUC representing a real aluminum foam. First, the error estimate on the computed dynamic thermal permeability is investigated for the cases of the canonical geometries having reference solutions. Second, the solution convergence is studied for the case of the nontrivial geometric PUC configuration. Finally, the computed dynamic thermal permeability of the PUC configuration is compared to experimental measurements on a real aluminum foam with a view to validating the proposed method.

\section{A. Error estimation}

Figure 4 compares analytical reference solutions ${ }^{27,29-31}$ and random walk (RW) simulations in terms of the dynamic thermal permeability for specific cross-sectional shapes of uniform pores: (a) slit with $1 \mathrm{~mm}$ width, (b) circle with $1 \mathrm{~mm}$ radius, (c) square with $\sqrt{2} \mathrm{~mm}$ side, and (d) equilateral triangle with $\sqrt{3} \mathrm{~mm}$ side. In order to validate the threedimensional implementation of the random walk simulation method, these shapes have been extruded, see Fig. 3. In these examples, for each simulation, the normalized trapping distance $\delta=t_{d} / R$ is set to $10^{-5}$, and the number of random walks to $500000, R$ being the circumscribed radius of the porous structure. Random walk simulation points represent the average of three distinctive random walk simulations. Simulating 500000 random walks with a $10^{-5}$ normalized trapping distance and computing dynamic thermal permeability values for 60 different frequencies required approximately between 90 min (three-dimensional slits made of 4 facets) and $210 \mathrm{~min}$ (circular pore made of 720 facets) with an Intel Pentium 4, 3.2 GHz. As one can note in Fig. 4, the random walk simulation points fall closely on the analytical reference curves. The relative differences between analytical and numerical solutions are quantified in Fig. 5. As an example, for a 500000 random walk simulation with a $10^{-5}$ normalized trapping distance, the error on the computed dynamic 
TABLE II. Regression coefficients for the evaluation of the mean error $\varepsilon$ on $\widetilde{k}_{\mathrm{eq}}^{\prime}(\omega)$ [in modulus $(\mathrm{Ab})$, real part $(\mathrm{Re})$, and imaginary part (Im)] as a function of the number of random walks $N(10000<N<1000000)$ for various normalized trapping distances $\delta$.

\begin{tabular}{|c|c|c|c|c|c|c|c|c|c|c|}
\hline & \multirow[b]{2}{*}{$\delta$} & \multicolumn{3}{|c|}{$\mathrm{Ab}$} & \multicolumn{3}{|c|}{$\mathrm{Re}$} & \multicolumn{3}{|c|}{$\mathrm{Im}$} \\
\hline & & $a$ & $b(\%)$ & $|r|$ & $a$ & $b(\%)$ & $|r|$ & $a$ & $b(\%)$ & $|r|$ \\
\hline \multirow[t]{9}{*}{ slit } & $10^{-2}$ & 0.02 & -180 & 0.26 & -0.07 & -140 & 0.84 & 0.00 & -150 & 0.02 \\
\hline & $10^{-3}$ & -0.06 & -226 & 0.24 & -0.22 & -126 & 0.80 & -0.11 & -177 & 0.28 \\
\hline & $10^{-4}$ & -0.40 & -79 & 0.99 & -0.43 & -32 & 0.96 & -0.68 & 71 & 0.93 \\
\hline & $10^{-5}$ & -0.52 & -20 & 0.92 & -0.54 & 22 & 0.92 & -0.40 & -62 & 0.74 \\
\hline & $10^{-6}$ & -0.55 & -16 & 0.88 & -0.56 & 25 & 0.92 & -0.45 & -41 & 0.74 \\
\hline & $10^{-7}$ & -0.59 & 17 & 0.86 & -0.50 & 0 & 0.90 & -0.72 & 99 & 0.85 \\
\hline & $10^{-8}$ & -0.51 & -13 & 0.72 & -0.47 & -3 & 0.82 & -0.48 & -4 & 0.65 \\
\hline & $10^{-9}$ & -0.13 & -156 & 0.64 & -0.15 & -134 & 0.71 & -0.11 & -140 & 0.54 \\
\hline & $10^{-10}$ & -0.01 & -117 & 0.15 & -0.01 & -102 & 0.28 & -0.01 & -87 & 0.35 \\
\hline \multirow[t]{9}{*}{ circle } & $10^{-2}$ & -0.01 & -174 & 0.29 & -0.03 & -174 & 0.57 & -0.02 & -161 & 0.28 \\
\hline & $10^{-3}$ & -0.13 & -209 & 0.34 & -0.41 & -40 & 0.90 & -0.23 & -136 & 0.49 \\
\hline & $10^{-4}$ & -0.33 & -144 & 0.76 & -0.48 & -25 & 0.93 & -0.37 & -101 & 0.68 \\
\hline & $10^{-5}$ & -0.42 & -94 & 0.83 & -0.43 & -53 & 0.90 & -0.48 & -51 & 0.92 \\
\hline & $10^{-6}$ & -0.36 & -103 & 0.77 & -0.40 & -58 & 0.87 & -0.43 & -51 & 0.70 \\
\hline & $10^{-7}$ & -0.30 & -148 & 0.62 & -0.41 & -55 & 0.90 & -0.47 & -44 & 0.79 \\
\hline & $10^{-8}$ & -0.55 & -17 & 0.80 & -0.53 & 9 & 0.94 & -0.43 & -58 & 0.79 \\
\hline & $10^{-9}$ & 0.00 & -227 & 0.00 & -0.08 & -170 & 0.44 & 0.07 & -243 & 0.24 \\
\hline & $10^{-10}$ & 0.01 & -125 & 0.41 & 0.02 & -121 & 0.64 & 0.00 & -99 & 0.14 \\
\hline \multirow[t]{9}{*}{ square } & $10^{-2}$ & -0.01 & -163 & 0.20 & -0.03 & -160 & 0.54 & -0.01 & -152 & 0.12 \\
\hline & $10^{-3}$ & -0.19 & -169 & 0.52 & -0.34 & -67 & 0.94 & -0.32 & -77 & 0.69 \\
\hline & $10^{-4}$ & -0.60 & 35 & 0.99 & -0.54 & 30 & 0.98 & -0.61 & 63 & 0.94 \\
\hline & $10^{-5}$ & -0.62 & 24 & 0.95 & -0.51 & 12 & 0.98 & -0.61 & 38 & 0.88 \\
\hline & $10^{-6}$ & -0.58 & 9 & 0.83 & -0.60 & 54 & 0.90 & -0.64 & 56 & 0.78 \\
\hline & $10^{-7}$ & -0.47 & -50 & 0.82 & -0.45 & -22 & 0.95 & -0.53 & -2 & 0.81 \\
\hline & $10^{-8}$ & -0.50 & -45 & 0.87 & -0.55 & 23 & 0.96 & -0.32 & -114 & 0.65 \\
\hline & $10^{-9}$ & 0.09 & -278 & 0.59 & -0.01 & -209 & 0.08 & 0.15 & -292 & 0.74 \\
\hline & $10^{-10}$ & 0.00 & -124 & 0.07 & -0.01 & -109 & 0.20 & 0.01 & -106 & 0.26 \\
\hline \multirow[t]{9}{*}{ triangle } & $10^{-2}$ & -0.02 & -139 & 0.50 & -0.04 & -143 & 0.77 & -0.02 & -133 & 0.22 \\
\hline & $10^{-3}$ & -0.19 & -150 & 0.50 & -0.35 & -59 & 0.90 & -0.26 & -96 & 0.59 \\
\hline & $10^{-4}$ & -0.35 & -117 & 0.69 & -0.37 & -68 & 0.92 & -0.30 & -116 & 0.63 \\
\hline & $10^{-5}$ & -0.61 & 23 & 0.82 & -0.65 & 83 & 0.97 & -0.58 & 30 & 0.75 \\
\hline & $10^{-6}$ & -0.41 & -75 & 0.90 & -0.40 & -47 & 0.90 & -0.38 & -71 & 0.81 \\
\hline & $10^{-7}$ & -0.28 & -143 & 0.74 & -0.42 & -38 & 0.98 & -0.37 & -71 & 0.78 \\
\hline & $10^{-8}$ & -0.63 & 38 & 0.85 & -0.52 & 16 & 0.88 & -0.47 & -25 & 0.79 \\
\hline & $10^{-9}$ & -0.07 & -184 & 0.38 & -0.10 & -155 & 0.54 & -0.11 & -142 & 0.66 \\
\hline & $10^{-10}$ & 0.02 & -131 & 0.49 & 0.03 & -129 & 0.60 & 0.01 & -106 & 0.36 \\
\hline
\end{tabular}

thermal permeability is estimated to be less than $(0.3 \pm 2) \%$ on the imaginary parts and less than $(0.2 \pm 5) \%$ on the modulus.

Now the influence of the trapping distance is investigated. For each cross-sectional shape, the normalized trapping distance $\delta$ is successively fixed to $10^{-2}, 10^{-3}, \ldots, 10^{-9}$, $10^{-10}$ and the number of random walks $N$ varies accordingly to the sequence $10000,20000,50000,70000,100000$, 200 000, 500 000, 700 000, 1000 000. Each case characterized by a different triplet (geometric configuration, $\delta N$ ) is simulated three times so that the dispersion of the results can also be determined. Four geometric configurations, nine normalized trapping distances, and $9 \times 3$ random walk simulations have been tested, for a total number of 972 numerical experiments. Simulation results are then processed in order to evaluate the mean error $\varepsilon$ compared to analytical reference solutions (average of the relative errors over 60 points) and the average of the standard deviations of the numerical results $\Delta \varepsilon$ (a standard deviation results from three points, and $\Delta \varepsilon$ is the average of 60 standard deviations). For each geometrical configuration and each normalized trapping distance, it is thus possible to determine coefficients $a, b$, and $r$, ( $r$ is the linear correlation coefficient) of the straight line of the form given by Eq. (44) and to minimize the squared differences with $9 \times 3$ random walk simulation points. These coefficients are reported in Tables II and III for the mean errors and standard deviations, respectively. The real part, imaginary part, and modulus of the dynamic thermal permeabilities are examined separately. The best correlation coefficients in in Table II and absolute values are generally higher than 0.9 (for the slits, the square, and the circle) and systematically higher than 0.8 ( 0.81 for the triangle), which means that a law of the form of Eq. (44) enables one to correlate the decreasing of the error logarithm with the num- 
TABLE III. Regression coefficients for the evaluation of the mean standard deviation $\Delta \varepsilon$ on $\widetilde{k}_{\mathrm{eq}}^{\prime}(\omega)$ [in modulus (Ab), real part (Re), and imaginary part (Im)] as a function of the number of random walks $N(10000<N<1000000)$ for various normalized trapping distances $\delta$.

\begin{tabular}{|c|c|c|c|c|c|c|c|c|c|c|}
\hline & \multirow[b]{2}{*}{$\delta$} & \multicolumn{3}{|c|}{$\mathrm{Ab}$} & \multicolumn{3}{|c|}{$\operatorname{Re}$} & \multicolumn{3}{|c|}{$\mathrm{Im}$} \\
\hline & & $a$ & $b(\%)$ & $|r|$ & $a$ & $b(\%)$ & $|r|$ & $a$ & $b(\%)$ & $|r|$ \\
\hline \multirow[t]{9}{*}{ slit } & $10^{-2}$ & -0.58 & 105 & 0.78 & -0.57 & 97 & 0.79 & -0.54 & 50 & 0.91 \\
\hline & $10^{-3}$ & -0.39 & 20 & 0.70 & -0.39 & 18 & 0.72 & -0.39 & -16 & 0.79 \\
\hline & $10^{-4}$ & -0.43 & 46 & 0.75 & -0.44 & 45 & 0.76 & -0.46 & 14 & 0.82 \\
\hline & $10^{-5}$ & -0.70 & 182 & 0.90 & -0.70 & 175 & 0.90 & -0.63 & 103 & 0.93 \\
\hline & $10^{-6}$ & -0.41 & 36 & 0.81 & -0.41 & 36 & 0.82 & -0.45 & 14 & 0.87 \\
\hline & $10^{-7}$ & -0.59 & 124 & 0.93 & -0.58 & 117 & 0.94 & -0.54 & 60 & 0.96 \\
\hline & $10^{-8}$ & -0.46 & 64 & 0.83 & -0.45 & 57 & 0.83 & -0.43 & 5 & 0.85 \\
\hline & $10^{-9}$ & -0.31 & -33 & 0.55 & -0.32 & -32 & 0.57 & -0.38 & -32 & 0.73 \\
\hline & $10^{-10}$ & -0.51 & 105 & 0.93 & -0.51 & 99 & 0.93 & -0.53 & 64 & 0.93 \\
\hline \multirow[t]{9}{*}{ circle } & $10^{-2}$ & -0.35 & -1 & 0.74 & -0.36 & -1 & 0.75 & -0.38 & -24 & 0.81 \\
\hline & $10^{-3}$ & -0.57 & 114 & 0.87 & -0.57 & 107 & 0.88 & -0.50 & 40 & 0.93 \\
\hline & $10^{-4}$ & -0.65 & 148 & 0.94 & -0.64 & 141 & 0.94 & -0.61 & 91 & 0.97 \\
\hline & $10^{-5}$ & -0.53 & 117 & 0.87 & -0.54 & 115 & 0.87 & -0.52 & 68 & 0.88 \\
\hline & $10^{-6}$ & -0.69 & 182 & 0.93 & -0.68 & 175 & 0.93 & -0.70 & 146 & 0.95 \\
\hline & $10^{-7}$ & -0.57 & 108 & 0.87 & -0.56 & 102 & 0.89 & -0.54 & 55 & 0.95 \\
\hline & $10^{-8}$ & -0.40 & 41 & 0.71 & -0.41 & 40 & 0.73 & -0.45 & 27 & 0.84 \\
\hline & $10^{-9}$ & -0.59 & 143 & 0.97 & -0.59 & 137 & 0.97 & -0.57 & 89 & 0.95 \\
\hline & $10^{-10}$ & -0.65 & 155 & 0.88 & -0.64 & 149 & 0.89 & -0.60 & 98 & 0.96 \\
\hline \multirow[t]{9}{*}{ square } & $10^{-2}$ & -0.51 & 65 & 0.80 & -0.51 & 62 & 0.81 & -0.51 & 33 & 0.87 \\
\hline & $10^{-3}$ & -0.68 & 154 & 0.85 & -0.67 & 143 & 0.86 & -0.52 & 42 & 0.89 \\
\hline & $10^{-4}$ & -0.42 & 30 & 0.90 & -0.42 & 27 & 0.91 & -0.40 & -16 & 0.92 \\
\hline & $10^{-5}$ & -0.40 & 31 & 0.90 & -0.40 & 28 & 0.91 & -0.38 & -20 & 0.88 \\
\hline & $10^{-6}$ & -0.48 & 59 & 0.85 & -0.48 & 55 & 0.85 & -0.51 & 37 & 0.90 \\
\hline & $10^{-7}$ & -0.68 & 153 & 0.96 & -0.67 & 146 & 0.96 & -0.62 & 86 & 0.95 \\
\hline & $10^{-8}$ & -0.42 & 24 & 0.78 & -0.42 & 23 & 0.80 & -0.46 & 7 & 0.87 \\
\hline & $10^{-9}$ & -0.62 & 131 & 0.86 & -0.62 & 122 & 0.87 & -0.56 & 65 & 0.90 \\
\hline & $10^{-10}$ & -0.46 & 59 & 0.81 & -0.46 & 54 & 0.82 & -0.41 & -3 & 0.87 \\
\hline \multirow[t]{9}{*}{ triangle } & $10^{-2}$ & -0.46 & 25 & 0.78 & -0.46 & 23 & 0.79 & -0.51 & 12 & 0.85 \\
\hline & $10^{-3}$ & -0.50 & 45 & 0.92 & -0.50 & 43 & 0.93 & -0.52 & 17 & 0.95 \\
\hline & $10^{-4}$ & -0.58 & 89 & 0.93 & -0.57 & 82 & 0.94 & -0.51 & 18 & 0.92 \\
\hline & $10^{-5}$ & -0.62 & 98 & 0.83 & -0.63 & 97 & 0.84 & -0.65 & 76 & 0.87 \\
\hline & $10^{-6}$ & -0.40 & -3 & 0.75 & -0.41 & -6 & 0.77 & -0.36 & -58 & 0.84 \\
\hline & $10^{-7}$ & -0.50 & 26 & 0.76 & -0.49 & 18 & 0.77 & -0.44 & -36 & 0.77 \\
\hline & $10^{-8}$ & -0.32 & -63 & 0.87 & -0.33 & -62 & 0.89 & -0.42 & -45 & 0.96 \\
\hline & $10^{-9}$ & -0.47 & 32 & 0.85 & -0.48 & 32 & 0.87 & -0.52 & 21 & 0.94 \\
\hline & $10^{-10}$ & -0.72 & 158 & 0.95 & -0.70 & 145 & 0.95 & -0.59 & 56 & 0.95 \\
\hline
\end{tabular}

ber of random walks, with a relatively high degree of confidence. Then, for each geometrical configuration, the higher correlation coefficients are associated with their corresponding normalized trapping distances. This means that, for each geometrical configuration, an adequate normalized trapping distance value is known, ensuring that the numerical solution converges to the reference solution according to a law of the form of Eq. (44). Furthermore, note that the correlation coefficients of Table III associated with the mentioned normalized trapping distances also correspond to a satisfying degree of confidence $(|r|>0.75)$. Consequently, for each specific shape, the dynamic thermal permeability might then be given with error $\varepsilon\left(a_{\varepsilon}, b_{\varepsilon}\right)$ and standard deviation $\Delta \varepsilon\left(a_{\Delta \varepsilon}, b_{\Delta \varepsilon}\right)$ estimates, where coefficients $a_{\varepsilon}, b_{\varepsilon}, a_{\Delta \varepsilon}$, and $b_{\Delta \varepsilon}$ are the shaded values reported in Tables II and III.

From the previous results, Table I summarizes the adequate trapping distances for the four canonical cross-section shapes. Table I suggests the following geometrical interpretation.
- The slit and the square are two specific configurations of the same geometry, the rectangle. This geometry essentially shows plane surfaces to the particle.

- The circle is modeled as a regular polygon of order $n=360$. The angle between two noncoplanar facets of the polygon is obtuse.

- The triangle is also a regular polygon, of order $n=3$. The angle formed between two noncoplanar facets is acute.

From this simple geometrical interpretation, it is suggested that the normalized trapping distance to use in a random walk simulation is as follows.

- $10^{-4}$ when the wet surfaces of the geometry are essentially plane;

- $10^{-5}$ when the wet surfaces of the geometry are essentially concave with obtuse angles;

- $10^{-6}$ when the wet surfaces of the geometry are essentially concave with acute angles. 
This attempt to interpret the trapping distance as a function of the geometry seems to be in accordance with the following physical representation. A relatively large normalized trapping distance increases the trapping risk before the particle had probed the fluid concavities of the porous space. Therefore, as the angle formed between two planes describing a fluid concavity tends to zero, the normalized trapping distance must also tend to zero in order to prevent the particle from being trapped before having probed the overall geometry.

\section{B. Solution convergence}

Here, the solution convergence is studied on a nontrivial PUC geometry as the one shown in Fig. 2. Figure 6 presents on a logarithmic scale the evolution of $L_{2}$ and $L_{\infty}$ norms with the number of random walks $N$ for three different normalized trapping distances $\delta: 10^{-4}, 10^{-5}$, and $10^{-6}$. Each point results from the averaging of three random walk simulation tests. A number of $11 \times 6 \times 3=198$ random walk simulations have been carried out to plot this graph (results obtained in approximately $4 \mathrm{~h}$ thanks to a serial PC cluster). A linear regression has been computed from the overall random walk simulation results using a function having the form of Eq. (44) and compared to convergence data. The linear regression line is obtained with a good correlation coefficient, $|r|$ $=0.95$. Tendency mentioned in Eq. (44) is thus reasonably verified and can be quantified with $a=-1.39$ and $b=3.42$. Typically, increasing the number of random walks by an order of magnitude divides by 25 the relative difference between two runs. For a number of 50000 random walks, the relative error is equal to $0.077 \%$; it is thus not necessary to increase significantly the number of random walks per simulation. It is the matter of a compromise between the computing time and desired precision. Furthermore, the $L_{2}\left(L_{\infty}\right)$ random walk simulation points are generally positioned above (below) the regression line. This confirms that $L_{\infty}$ norm is actually more restrictive than $L_{2}$ norm.

Therefore, one can reasonably conclude to the stability of the numerical solution computed by the random walk simulation method for the three-dimensional PUC: the sum of the relative differences on a large frequency range, between the computed response for a number of random walks equal to $N+\Delta N$ and the computed response for a number of random walks equal to $N$, is small and decreases monotonically in average, whatever the norm considered.

As a summary, it has been shown that

- practically, monotonic convergence is observed for the random walk simulation method according to a logarithmic law;

- for the 3D PUC, relative variations with the number of random walks is predictable and a good compromise between computational time and precision is obtained with 50000 random walks.

\section{Comparison with experimental data}

Here, the random walk method is used to compute the dynamic thermal permeability of an idealized PUC of a real aluminum foam. The reconstruction method of the PUC by $\mathrm{X}$-ray computed microtomography was presented elsewhere by the authors. ${ }^{26}$ The aluminum foam studied is the 40 pores per linear inch (PPI) open-cell aluminum foam manufactured by ERG Aerospace. The reconstructed PUC is the one shown in Fig. 2. The computations were run and averaged over 50000 random walks. The normalized trapping distance was taken to be $10^{-5}$ (the wet surfaces of the PUC are essentially plane or concave with obtuse angles). Under these conditions, a simulation requires less than half an hour on a CPU Intel P4, 3.2 GHz, 2 Gbyte RAM. Figure 7 shows the computed equivalent dynamic thermal permeability $\widetilde{k}_{\mathrm{eq}}^{\prime}(\omega)$ converted into the equivalent dynamic bulk modulus $K_{\text {eq }}(\omega)$ normalized by the adiabatic bulk modulus $K_{a}$ and compared to impedance tube measurements. It has been shown by Lafarge et $a{ }^{7}{ }^{7}$ that the dynamic bulk modulus $\widetilde{K}_{\text {eq }}(\omega)$ of an equivalent fluid medium can be linked to its dynamic thermal permeability $\widetilde{k}_{\mathrm{eq}}^{\prime}(\omega)$ according to the following expression:

$$
\frac{K_{a}}{\tilde{K}_{\mathrm{eq}}(\omega)}=\gamma+(\gamma-1) \frac{j \omega}{\nu^{\prime}} \tilde{k}_{\mathrm{eq}}^{\prime}(\omega),
$$

where $K_{a}=\gamma P_{0}$ is the adiabatic bulk modulus, $\gamma$ is the heat capacity ratio, and $P_{0}$ the atmospheric pressure. The macroscopic measurements (dots) were obtained using a $44.4 \mathrm{~mm}$ impedance tube with the two-cavity technique. ${ }^{32}$ Good agreement is observed between the simulations and the measurements, especially at the higher frequencies. As one can note, the numerical computations predict a transition frequency around $16 \mathrm{~Hz}$. This very low transition frequency is due to the large cells of the studied 40 pore/in. aluminum foam (pore diameter greater than $2 \mathrm{~mm}$, see Ref. 26). With the used experimental setup, it was not possible to obtain higher accuracy in the low frequency range; however, the experimental results in the measurable frequency range $(100-1000 \mathrm{~Hz})$ are in good accordance with the predicted post-transition and asymptotic adiabatic regimes.

\section{SUMMARY AND CONCLUSIONS}

We have applied a numerical method for the determination of the equivalent dynamic thermal permeability $\widetilde{k}_{\text {eq }}^{\prime}(\omega)$ of a 3D PUC of porous media. This method is based on a Brownian motion simulation technique using a random walk algorithm. It can be applied to porous media for which a periodic unit cell is identified by means of computer microtomography. The interest of this method is that the simulation of random walks is independent of frequency. This means that, once the random walks have been simulated for a given geometric configuration, the thermal permeability can be computed whatever the frequency, with the same computational cost, showing a relatively fast execution time. A 3D PUC of open cell aluminum foam provides a numerical example showing convergence in terms of $L_{2}$ and $L_{\infty}$ norms. Typically, increasing the number of random walks by an order of magnitude divides by 25 the relative error on the prediction. The numerical method has also been applied to specific cross-sectional shapes of uniform pores having reference solutions. In this case, the computed dynamic ther- 
mal permeabilities are found to be in excellent agreement with the reference solutions. The numerical solution is very accurate when the data represent averages over 50000 random walk realizations, with a normalized trapping distance $\delta$ fixed to $10^{-5}$ and the mean error inferior to $0.25 \%$ in modulus for all models of uniform pores. Note that $\delta$ needs to be refined if the geometrical configuration shows concave surfaces with very acute angles, in order to prevent the particle from being trapped before having probed the overall geometry. To demonstrate the application of our results, we have compared the numerical simulations to experimental data obtained from impedance tube measurements of an open cell aluminum foam. For the sample studied here, numerical simulations provided a good estimate of the dynamic bulk modulus without any adjustable parameter. However, due to its large cell size, it was difficult to measure the low frequency behavior of the aluminum foam. Further research is required on commonly used open cell foams in acoustics, including fully measurable thermal transition frequencies (i.e., pore radius $\sim 0.1 \mathrm{~mm}$ ).

\section{ACKNOWLEDGMENTS}

This work was supported by Alcan, CQRDA, REGAL, FQRNT, NSERC, Auto21, and Région Rhône-Alpes. C. Perrot would like to thank R. Bouchard, L.-M. Raynauld, F. Jackson, and S. Allen for their helpful contributions in the development of the numerical programs. Finally, computations were performed at the Centre for Computational Science at the Université de Sherbrooke whose support is gratefully acknowledged.

\section{APPENDIX: REGRESSION COEFFICIENTS}

Tables II and III present the results of the linear regressions over the computed dynamic thermal permeabilities and the reference solutions where $a$ and $b$ are the coefficients of Eq. (44) and $r$ the correlation coefficient.

\footnotetext{
${ }^{1}$ A. Cummings and S. P. Beadle, J. Sound Vib. 175, 115 (1994).

${ }^{2}$ R. T. Muehleisen, C. W. Beamer, and B. D. Tinianov, J. Acoust. Soc. Am 117, 536 (2005).
}

${ }^{3}$ D. L. Johnson, J. Koplik, and R. Dashen, J. Fluid Mech. 176, 379 (1987). ${ }^{4}$ Y. Champoux and J. F. Allard, J. Appl. Phys. 70, 1975 (1991).

${ }^{5}$ J. F. Allard, Propagation of Sound in Porous Media: Modeling Sound Absorbing Materials, edited by Elsevier Applied Science (Elsevier, New York, 1993), p. 284.

${ }^{6}$ S. R. Pride, F. D. Morgan, and A. F. Gangi, Phys. Rev. B 47, 4964 (1993). ${ }^{7}$ D. Lafarge, P. Lemarinier, J. F. Allard, and V. Tarnow, J. Acoust. Soc. Am. 102, 1995 (1997).

${ }^{8}$ A. Craggs and J. G. Hildebrandt, J. Sound Vib. 92, 321 (1984).

${ }^{9}$ M. Firdaouss, J.-L. Guermond, and D. Lafarge, Int. J. Eng. Sci. 36, 1035 (1998).

${ }^{10}$ A. Cortis, D. M. L. Smeulders, D. Lafarge, M. Firdaouss, and J.-L. Guermond, in Solid Mechanics and Its Applications, IUTAM Symposium on Theoretical and Numerical Methods in Continuum Mechanics of Porous Materials, University of Stuttgart, edited by W. Ehlers (Kluwer Academic, Dordrecht, 1999), pp. 187-192.

${ }^{11}$ K. Boomsma, D. Poulikakos, and Y. Ventikos, Int. J. Heat Fluid Flow 24, 825 (2003).

${ }^{12}$ A. Cortis, D. M. J. Smeulders, J.-L. Guermond, and D. Lafarge, Phys. Fluids 15, 1766 (2003).

${ }^{13}$ S. Gasser, F. Paun, and Y. Brechet, J. Acoust. Soc. Am. 117, 2090 (2005).

${ }^{14}$ M. Y. Zhou and P. Sheng, Phys. Rev. B 39, 12027 (1989).

${ }^{15}$ D. Lafarge, in Poromechanics II, Proceedings of the Second Biot Conference on Poromechanics, edited by J.-L. Auriault (Swets \& Zeitlinger, Grenoble, 2002), pp. 703-708.

${ }^{16}$ R. Feynman, R. Leighton, and M. Sands, The Feynman Lectures on Physics (Addison-Wesley, Reading, MA, 1971), Vol. 1, Chap. 6.

${ }^{17}$ S. Torquato, Appl. Phys. Lett. 55, 1847 (1989).

${ }^{18}$ D. A. Coker, J. Appl. Phys. 77, 955 (1994).

${ }^{19}$ J.-L. Auriault, L. Borne, and R. Chambon, J. Acoust. Soc. Am. 77, 1641 (1985).

${ }^{20}$ L. H. Zheng and Y. C. Chiew, J. Chem. Phys. 90, 322 (1989).

${ }^{21}$ N. Agmon, J. Chem. Phys. 81, 3644 (1984).

${ }^{22}$ C. Perrot, "Microstructure et Macro-Comportement Acoustique: Approche par Reconstruction d'une Cellule Élementaire Représentative," Ph.D. thesis, Université de Sherbrooke and INSA de Lyon, 2006 (available online at http://tel.archives-ouvertes.fr/tel-00123464).

${ }^{23}$ D. Lafarge (private communication).

${ }^{24}$ L. Pontryagin, A. Andronow, and A. Witt, Zh. Eksp. Teor. Fiz. 3, 172 (1933).

${ }^{25}$ S. Lifson and J. L. Jackson, J. Chem. Phys. 36, 2410 (1962).

${ }^{26}$ C. Perrot, R. Panneton, and X. Olny, J. Appl. Phys. 101, 113528 (2007).

${ }^{27}$ M. R. Stinson, J. Acoust. Soc. Am. 89, 550 (1991).

${ }^{28}$ G. Kirchhoff, Ann. Phys. Chem. 134, 177 (1868).

${ }^{29}$ K. Attenborough, J. Acoust. Soc. Am. 73, 785 (1983).

${ }^{30} \mathrm{C}$. Zwikker and C. W. Kosten, in Sound Absorbing Materials, edited by Elsevier Applied Sciences (Elsevier Applied Sciences, New York, 1949).

${ }^{31}$ M. R. Stinson and Y. Champoux, J. Acoust. Soc. Am. 91, 685 (1992).

${ }^{32}$ H. Utsuno, T. Tanaka, T. Fujikawa, and A. F. Seybert, J. Acoust. Soc. Am. 86, 637 (1989). 